\title{
Characterization of a Suite of 40 EST-derived Microsatellite Markers For Use in Sitka Spruce (Picea sitchensis (Bong.) Carr)
}

\author{
By S. W. A'HarA*) and J. E. CotTrell \\ Forest Research, Northern Research Station, Roslin, Midlothian, EH25 9SY, Scotland, UK.
}

(Received $4^{\text {th }}$ April 2006)

\begin{abstract}
This paper describes 40 novel, data-mined, polymorphic microsatellite loci for use in a QTL association study in Sitka spruce. Publicly available EST sequences of Picea in Genbank were searched in silico for simple sequence repeat (SSR) motifs, principally dinucleotide microsatellites, and PCR primers were designed to flank these regions. PCR amplification was carried out in the progeny of a full-sib family to test simple Mendelian inheritance. For further characterization, the amplification products of Sitka spruce material from unrelated trees were assessed to determine the potential of these loci for population genetic studies. These polymorphic markers therefore represent a valuable tool-kit both for establishing a molecular map of this species and for Picea genetic population studies.
\end{abstract}

Key words: Picea sitchensis, microsatellites, genetic map, marker aided selection

\section{Introduction}

Sitka spruce [Picea sitchensis (Bong.) Carr] is the primary commercial forestry species in the UK. Traditional tree breeding programmes have been successful in improving economically important traits but these are slow and expensive. A quantative trait loci (QTL) association study has been initiated by Forest Research, UK, to accelerate genetic improvement in Sitka spruce. QTL studies aim to associate performance in the field with DNA markers assessed in the laboratory and offer the possibility, in the future, of selecting trees with particular desirable traits without the need for expensive and protracted progeny trials.

Comprehensive field trials comprising 4,500 Sitka spruce clones have been set up, involving complete replication over three climatically contrasting sites. The 4,500 clones are sub-divided into 1,500 clones from each of three families about which prior knowledge of parental genetic quality is known. Each family consists of parents with contrasting phenotypes for the following economically important traits; wood density, straightness and branching. The trials were planted in spring 2005 in North Scotland (57.1302N, 3.5684W; Accumulated Temperature (AT) over $5{ }^{\circ} \mathrm{C}$ (monthly mean over 30 years $)=1100)$, South Wales $(51.6938 \mathrm{~N}, 4.8157 \mathrm{~W}$; $\mathrm{AT}=1450)$ and South England $(50.7706 \mathrm{~N}, 4.7807 \mathrm{~W}$; AT $=1800$ ). This will enable the robustness of marker/

*) Corresponding author: S. W. A'HARA. Fax: 0131-4455124. E-mail: Stuart.A'Hara@forestry.gsi.gov.uk trait association to be tested in three families both within, and between, three sites.

The recent advances in the process of genetic mapping and QTL association in spruce has been greatly assisted by the public release of expressed sequence tagged (EST) information, which has enabled DNA markers to be discovered with relative ease and reduced cost (see UJINO-IHARA et al., 2005 for details of spruce and pine EST databases). Highly polymorphic, co-dominant microsatellite markers are the markers of choice for genetic mapping studies as they not only allow the fusion of male and female maps in a pseudo-test cross mapping strategy, but also are likely to show a degree of transferability across species within a genus. (LIEWLAKSANEEYANAWIN et al., 2004; RUNGIS et al., 2004). Ultimately, this will enable the map position of putative QTLs or candidate genes for a particular trait to be compared across species.

SSR discovery from genomic libraries has proved problematic in conifers, with a low return for effort. (RAJORA et al., 2001; HoDGETTS et al., 2001). This is deemed to be because of the large size and repetitive nature of the conifer genome.

It was generally considered that EST derived microsatellite markers are less variable than genomic microsatellites (gSSRs) due to their position in coding regions and the assumption that they are therefore under a degree of selection pressure. Recent results in Picea, however, demonstrated only slightly lower levels of polymorphism in EST derived microsatellites (RUNGIS et al., 2004). Furthermore, EST derived microsatellites exhibit a lower number of null alleles than gSSRs (RUNGIS et al., 2004) making them more useful for population genetic studies. These considerations, in addition to their relative ease of discovery, make them high utility markers. In addition, by focussing efforts on dinucleotide repeats, which were shown to be a more polymorphic motif than tri- or tetra-nucleotides in Sitka spruce (RUNGIS et al., 2004), the possibility of finding useful polymorphic loci for the three mapping populations was maximised.

\section{Materials and Methods}

Picea EST sequences from $P$. glauca, $P$. sitchensis and $P$. sitchensis $\mathrm{x} P$. engelmannii publicly available in Genbank in November 2005 were downloaded and processed with the CAP3 software program (HUANG and MADAN (1999); http://deepc2.psi.iastate.edu/aat/cap/cap.html) 
Table 1. - Primer pairs developed from Picea ESTs for amplification of SSR markers. Locus name, Genbank accession number, allele size range, SSR motif and best protein match are presented.

\begin{tabular}{|c|c|c|c|c|c|}
\hline Locus & Accession & Primer pair & $\begin{array}{l}\text { Allele } \\
\text { size range }\end{array}$ & Motif & $\begin{array}{l}\text { Best protein } \\
\text { match }\end{array}$ \\
\hline s\$18 & 40766244 & $\begin{array}{l}\text { F: *M13-GTGCCGTGAACCACATTAAC } \\
\text { R: GCGTACATGAAAACCTGCAT }\end{array}$ & $248-282$ & $(\mathrm{~A})_{19}(\mathrm{TA})_{6}$ & unknown \\
\hline s\$19 & 40767122 & $\begin{array}{l}\text { F: M13-GAGAAAACCTGGCCTCTAGAAA } \\
\text { R: } \quad \text { GGGACCATCTCCTAGACACG }\end{array}$ & $218-226$ & $(\mathrm{AT})_{6}$ & $\begin{array}{l}\text { NP } 910052 \text { putative Hsp } 70 \text { binding protein [Oryza sativa (japonica } \\
\text { cultivar-group)]. }\end{array}$ \\
\hline s\$20 & 40775728 & $\begin{array}{l}\text { F: M13-TAACCGATGCCTCTCACAGC } \\
\text { R: } \quad \text { TGATGCATCCATCCTTGTTG }\end{array}$ & 196-212 & $(\mathrm{AT})_{6}$ & $\begin{array}{l}\text { AAB70558 putative protein tyrosine phosphatase homologue } \\
\text { [Homo sapiens]. }\end{array}$ \\
\hline$s \$ 21$ & 40779303 & $\begin{array}{l}\text { F: } \\
\text { R: }\end{array}$ & $180-226$ & $(A)_{8}(C A)_{5}(T A)_{10}$ & CAA31594 D-34 Lea protein [Gossypium hirsutum]. \\
\hline s\$22 & 49016032 & $\begin{array}{l}\text { F: M13-CACTCCAAGCAAGAATCTCCA } \\
\text { R: } \quad \text { GACATCTCAGGCGATTCCAT }\end{array}$ & $246-250$ & $(\mathrm{AT})_{5}(\mathrm{CT})(\mathrm{AT})(\mathrm{AT})_{3}$ & $\begin{array}{l}\text { AAZ21700 NADH Dehydrogenase I Chain C } \\
\text { [Candidatus Pelagibacter ubique] }\end{array}$ \\
\hline ss23 & 49017151 & $\begin{array}{l}\text { F: } \\
\text { R: } 13-\text { GCTCCTTCAGATCAGCGAAC } \\
\text { AGAGGCAAGTTCAGGGATT }\end{array}$ & $196-210$ & $(\overline{A T T})_{7}(\mathrm{~T})_{5}$ & YP_224083 L [Taro vein chlorosis virus]. \\
\hline sS24 & 49024553 & $\begin{array}{l}\text { F: M13-CCTTAACCCCTACTGTCAATGC } \\
\text { R: CGGCTTGTGGGATAACAAG }\end{array}$ & $258-282$ & $(T A)_{4}(A)_{3}(T A)_{4}$ & $\begin{array}{l}\mathrm{XP} 670126 \text { hypothetical protein PB } 400116.00 .0 \\
\text { [Plasmodium berghei strain] }\end{array}$ \\
\hline$s \$ 25$ & 49037755 & $\begin{array}{l}\text { F: M13-AACATCCACCCATTCAAAGC } \\
\text { R: } \quad \text { GTGCATGCGAATCCCTAAAC }\end{array}$ & $218-264$ & $(\mathrm{AT})_{13}$ & ABA96147 expressed protein [Oryza sativa (japonica cultivar-group)]. \\
\hline $\operatorname{ss} 26$ & 49041358 & $\begin{array}{l}\text { F: } \\
\text { R: } 13-\text { AGGTTGGAAACCCTGTGATG } \\
\text { ATTCGGATTGCGGTTCTGTA }\end{array}$ & $198-218$ & $(T A)_{8}$ & BAD79321 hypothetical protein [Synechococcus elongatus PCC 6301]. \\
\hline S\$27 & 49041823 & $\begin{array}{l}\text { F: M13-TGACGTTATTAATGGCGTTTG } \\
\text { R: TTTGAGGGGAGATCTTGTGG }\end{array}$ & 176-204 & $(T A)_{10}$ & YP_073752 U11 [Human herpesvirus 7]. \\
\hline ss 28 & 49043234 & $\begin{array}{l}\text { F: M13-GCTGAGGCAATGATGTCAAC } \\
\text { R: } \quad \text { GACATCTGTTCATTGCTCTACTTG }\end{array}$ & $256-284$ & $(\mathrm{TA})_{13}$ & $\begin{array}{l}\text { ABA93984 oxidoreductase, short chain dehydrogenase/reductase } \\
\text { family [[Oryza sativa (japonica cultivar-group)]. }\end{array}$ \\
\hline $\operatorname{ss} 29$ & 49043836 & $\begin{array}{l}\text { F: } \\
\text { R: }\end{array}$ & $254-286$ & $(\mathrm{TA})_{12}$ & GSU0558 hypothetical protein [Geobacter sulfurreducens PCA]. \\
\hline $\operatorname{ss} 30$ & 49044204 & $\begin{array}{l}\text { F: } \\
\text { R: }\end{array}$ & $270-294$ & $(\mathrm{TA})_{\mathrm{g}}$ & unknown \\
\hline SS31 & 49045444 & $\begin{array}{l}\text { F: M13-CGGAGTCTTGGTGGAACATT } \\
\text { R: } \quad \text { GGAGCAATCCCTCTTCTTCC }\end{array}$ & $144-190$ & $(T A)_{11}$ & unknown \\
\hline SS32 32 & 49045765 & $\begin{array}{l}\text { F: M13-CTGAAGCAAACACGACAAGC } \\
\text { R: } \quad \text { CCACATGCCTGCACTATCAT }\end{array}$ & $242-262$ & $\begin{array}{l}(T A)_{4}(A)(T A)_{9} \\
(C A)_{4}(T A)_{6}\end{array}$ & NP_849973 metal ion binding [Arabidopsis thaliana]. \\
\hline SS33 & 49046524 & $\begin{array}{l}\text { F: } \\
\text { M13-ACCTCAAGGGGCTACACTGA } \\
\text { R: } \quad \text { TAACGAGCCTGGTGTGTTTG }\end{array}$ & $188-194$ & $(T A)_{8}$ & unknown \\
\hline s\$34 & 49047398 & $\begin{array}{l}\text { F: M13-TTGCCCTGAGTAGGGTACAA } \\
\text { R: } \quad \text { AGCATTTGCTTGTTTCGTGA }\end{array}$ & $260-262$ & $(\mathrm{TA})_{3}(\mathrm{TG})_{3}(\mathrm{TC})(\mathrm{TA})_{4}$ & XP_640606 hypothetical protein DDB0204634 [Dictyostelium discoideum]. \\
\hline SS35 & 49047601 & $\begin{array}{l}\text { F: M13-TCCAATCCAAAACCGAAAAC } \\
\text { R: } \quad \text { GGCCTGTGTCTTTTCCATGT }\end{array}$ & $262-268$ & $(T A)_{8}$ & AAL39230 GH10154p [Drosophila melanogaster]. \\
\hline s\$36 & 49047619 & $\begin{array}{lr}\text { F: } & \text { M13-GTGTTCGAATCCCAGGAAGA } \\
\text { R: } & \text { TGCCCTGTGCGATGTTATAG }\end{array}$ & $232-238$ & $(G A)_{8}$ & $\mathrm{XP}_{-} 684896$ similar to LReO_3 [Danio rerio]. \\
\hline S\$37 & 49047962 & $\begin{array}{l}\text { F: M13-GGCCCCAAGATTGAAGAAGT } \\
\text { R: } \quad \text { TTTGGAATGCAATGCAGTTTAC }\end{array}$ & $224-226$ & $(T A)_{5}$ & XP_480282 putative RGH1 Oryza sativa (japonica cultivar-group) \\
\hline ss38 & 49048259 & $\begin{array}{l}\text { F: } \\
\text { M13-GAACGCAAGATGGGACACTT } \\
\text { R: } \quad \text { TGACCGTCTCTGTCCTTGTC }\end{array}$ & $206-221$ & $(\mathrm{GGA})_{6}$ & CAJ08228 calpain-like cysteine peptidase, putative; cysteine peptidas \\
\hline $\operatorname{ss} 39$ & 49048405 & 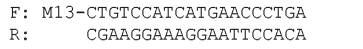 & $224-258$ & $(T A)_{10}$ & XP_473723 OSJNBa0086006.23 [Oryza sativa (japonica cultivar-group)]. \\
\hline SS40 & 49048492 & $\begin{array}{l}\text { F: M13-TCCCTCCTATACGGGAATGT } \\
\text { R: } \quad \text { GCGTACGTTAGTCGATTGGAA }\end{array}$ & $202-204$ & (СT) 6 & NP_196009 unknown protein [Arabidopsis thaliana]. \\
\hline SS41 & 49048842 & $\begin{array}{l}\text { F: M13-GCCAGAATGGTTTTCACGAG } \\
\text { R: } \quad \text { ATATCCTTGGGAGCGACCTT }\end{array}$ & $274-280$ & $(T A)_{6}(\mathrm{AA})(\mathrm{TA})_{4}$ & $\begin{array}{l}\text { BAD } 7127 \text { receptor protein kinase-like [Oryza sativa (japonica } \\
\text { cultivar-group)]. }\end{array}$ \\
\hline SS42 & 49049101 & $\begin{array}{lr}\text { F: } & \text { M13-TCGACCCTCTAACGTCTTGC } \\
\text { R: } & \text { TGAGAATCATCCCGAACTCC }\end{array}$ & $230-248$ & $(T A)_{6}$ & EAA04656 ENSANGP00000009427 [Anopheles gambiae str. PEST]. \\
\hline ss43 & 49049495 & $\begin{array}{l}\text { F: M13-ACAATGAGGATGAGGGCTTG } \\
\text { R: } \quad \text { CCCCTTTCGAACTGTGTCAT }\end{array}$ & $266-298$ & $(\mathrm{TA})_{16}$ & CAB46056 putative protein [Arabidopsis thaliana]. \\
\hline $\operatorname{sS} 44$ & 49049559 & $\begin{array}{l}\text { F: M13-CAAACGGAAGTCGAACCATT } \\
\text { R: } \quad \text { ATTTGTTTTCGGGTCTGCTG }\end{array}$ & $222-226$ & $(\mathrm{~A}) 10$ & unknown \\
\hline SS45 & 49135131 & $\begin{array}{l}\text { F: M13-AACACACGAGGGGATTGAAC } \\
\text { R: } \quad \text { ACCTGGCTTTCGGTATTGTG }\end{array}$ & $216-236$ & $(\mathrm{AT})_{\mathrm{B}}$ & unknown \\
\hline SS46 & 49137541 & $\begin{array}{l}\text { F: M13-GACAGGCACCCAGAACTGAT } \\
\text { R: CTGGAATTTACAATGCACAGGA }\end{array}$ & $232-288$ & $(\mathrm{AT})_{13}$ & unknown \\
\hline S\$47 & 69437955 & $\begin{array}{l}\text { F: } \\
\text { R: }\end{array}$ & $242-302$ & $(\mathrm{AT})_{14}$ & CAJ4359 Snakin-like cysteine rich protein [Phaseolus vulgaris]. \\
\hline $\operatorname{sS} 48$ & 70256787 & $\begin{array}{l}\text { F: M13-TCAAATCCAATCACGTACAACA } \\
\text { R: } \quad \text { CAACTTGAAGCCCCACTTGT }\end{array}$ & $256-278$ & $(\mathrm{AT})_{\mathrm{B}}$ & AAB 66560 anthocyanidin synthase [Callistephus chinensis]. \\
\hline SS49 & 70258880 & $\begin{array}{l}\text { F: M13-CAGTGCACGTCCAAACAAGT } \\
\text { R: } \quad \text { TGGCTGTTTGCTCATATTGC }\end{array}$ & $266-292$ & $(\mathrm{CT})_{16}$ & unknown \\
\hline s\$50 & 70260028 & $\begin{array}{l}\text { F: M1 3-TCAGAAGACGCAAACAATCCT } \\
\text { R: } \quad \text { TTCTTAGGAGGCAGCGGTTA }\end{array}$ & $164-178$ & $(\mathrm{AT})_{10}$ & AAC05682 germin-like protein [Oryza sativa]. \\
\hline ss51 & 70285403 & $\begin{array}{l}\text { F: M13-CAAAACACAACTTGCCCACA } \\
\text { R: } \quad \text { TGGAACACTTGGACTCCATT }\end{array}$ & $176-220$ & $(\mathrm{TA})_{11}$ & NP_565937 hypothetical protein [Arabidopsis thaliana]. \\
\hline ss52 & 70297371 & $\begin{array}{l}\text { F: } \\
\text { R13-AAGAGTCGCTCAAGGGCATA } \\
\text { R: } \quad \text { CATCTGAGAGGTCGCCATTT }\end{array}$ & $196-218$ & $(\mathrm{TA})_{10}$ & AAT80888 chloroplast chaperonin 21 [Vitis vinifera]. \\
\hline ss53 & 70318999 & $\begin{array}{l}\text { F: M13-CCGACGGTAGTTCCTTTCAA } \\
\text { R: CCGGTGCTAAATCCTTGTGT }\end{array}$ & $198-218$ & $(T A)_{B}$ & AAL13436 anaphase promoting complex subunit 11 [Arabidopsis thaliana] \\
\hline SS54 & 70346839 & $\begin{array}{l}\text { F: M13-AAGCATGATCAGTGGATAGCA } \\
\text { R: } \quad \text { AATTGAGGCAATTGGTGAGG }\end{array}$ & $220-240$ & $(\mathrm{GA})_{10}$ & unknown \\
\hline $\operatorname{ss5} 5$ & 70349470 & $\begin{array}{l}\text { F: M13-GAATTTAATGGCAACCATACCC } \\
\text { R: } \quad \text { TGTTTTGACTCGGATCTCTCTC }\end{array}$ & $160-178$ & $(\mathrm{TA})_{12}$ & $\mathrm{XP}_{-} 474343$ OSJNBa0064G10.8 [Oryza sativa (japonica cultivar-group)]. \\
\hline $\operatorname{ss} 56$ & 70350346 & $\begin{array}{l}\text { F: M13-GAAAATCGCCGAAAGATCAC } \\
\text { R: } \quad \text { GCCAGCATTCACTTGACAGA }\end{array}$ & $248-264$ & $(T A)_{B}$ & CAA70101 L-lactate dehydrogenase [Lycopersicon esculentum]. \\
\hline SS57 & 70355804 & $\begin{array}{l}\text { F: M13-CATCGTACAGCCAATCTCCA } \\
\text { R: } \quad \text { GAGGTCGTCCCGACAGTAGT }\end{array}$ & $126-140$ & $(\mathrm{TA})_{10}$ & unknown \\
\hline
\end{tabular}

\section{* M13 tail: 5’-AGGGTTTTCCCAGTCACGACGTT-3’}

which aligns over-lapping sequences into "contigs". This "contiging" process allows any SSR length polymorphisms from different accessions to be readily highlighted in silico. Such polymorphic sequences were chosen preferentially for primer design, although some unique sequences containing a microsatellite motif were also used. The microsatellite repeat regions were identified by searching the CAP3 output files using the MREPS program (KOLPAKOV et al., 2003); http://bioinfo.lifl.fr/mreps/) which identifies repeat motifs. Primer sequences were then designed around the repeats using the PRIMER 3 program (ROZEN and SKALETSKY, 2000); http://frodo.wi.mit.edu/cgi-bin/ primer3/primer3_www.cgi) (Table 1). Primers were ordered from MWG Biotech, Germany. Forward primers were tailed with a M13 sequence which meant a considerable reduction in costs when screening primers.

DNA was extracted by freezing four finely chopped needles per sample in liquid nitrogen and grinding with two stainless steel balls $(4 \mathrm{~mm})$ in a $2 \mathrm{ml}$ Eppendorf tube using a Retsch MM300 mixer-mill. Once the needles were reduced to a fine powder, DNA was extracted 
using a Qiagen DNeasy kit (http://Qiagen.com) following the manufacturer's instructions.

PCR was carried out in a $10 \mu \mathrm{l}$ total volume containing 25-50 ng genomic DNA. The reaction mix consisted of $10 \mathrm{mM} \mathrm{KCl}, 10 \mathrm{mM}\left(\mathrm{NH}_{4}\right)_{2} \mathrm{SO}_{4}, 20 \mathrm{mM}$ Tris-HCl, $2 \mathrm{mM} \mathrm{MgSO}_{4}, 0.1 \%$ Triton X-100 at $\mathrm{pH} 8.8$ (New England Biolabs, UK) and $0.2 \mathrm{mM}$ of each dNTP, $0.3 \mathrm{mM}$ of M13-tailed forward primer, $0.3 \mathrm{mM}$ of reverse primer, $0.05 \mathrm{mM}$ of M13 oligo, labelled with either 700 or 800 fluorescent dye (MWG Biotech, Germany) and 1.0U Taq polymerase (New England Biolabs, UK). The PCR protocol consisted of an initial denaturation step of 3 mins at $94^{\circ} \mathrm{C}$, followed by 10 cycles of: $94^{\circ} \mathrm{C}$ for 30 secs, $57^{\circ} \mathrm{C}$ for $1 \mathrm{~min}$ and $72^{\circ} \mathrm{C}$ for $30 \mathrm{secs}$. This was followed by 28 cycles of: $94^{\circ} \mathrm{C}$ for $30 \mathrm{secs}, 55^{\circ} \mathrm{C}$ for 1 min then $72^{\circ} \mathrm{C}$ for 30 secs. A final elongation step of 6 mins at $72^{\circ} \mathrm{C}$ was then carried out.

The amplification products were electrophoresed through a $6 \%$ denaturing polyacrylamide gel on a Licor IR sequencer.

The number of alleles, effective number of alleles, observed and expected heterozygosities and inbreeding coefficient $(\mathrm{F})$ were calculated using the POPGENE program (YEH and BOYLE, 1997; http://www.ualberta.ca/ $\sim$ fyeh). The g-square test and the chi-square test were also applied to these data to test for Hardy-Weinberg Equilibrium.

Table 2. - Characterization and statistics of SSR markers in P. sitchensis in the progeny of a full-sib cross and in a set of unrelated samples.

\begin{tabular}{|c|c|c|c|c|c|c|c|c|c|c|c|}
\hline $\begin{array}{l}\stackrel{0}{2} \\
2 \\
0 \\
0 \\
\qquad\end{array}$ & 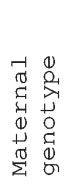 & 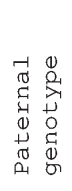 & 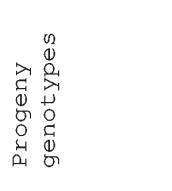 & 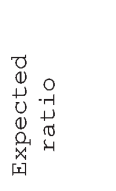 & $\begin{array}{ll}0 & \\
0 & 0 \\
0 & 0 \\
3 & -7 \\
0 & +1 \\
02 & 0 \\
0 & 4 \\
0 & \end{array}$ & ت્ & $\begin{array}{l}0 \\
0 \\
0 \\
0 \\
.4 \\
-1 \\
-1 \\
0 \\
0 \\
\dot{0} \\
z\end{array}$ & 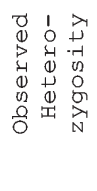 & 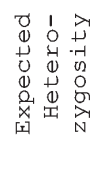 & 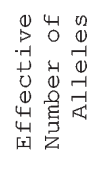 & [ᄃㅏㄴ \\
\hline SS18 & $\operatorname{lm}$ & 11 & $11: 1 \mathrm{~m}$ & $1: 1$ & $94: 93$ & 0.0 & 20 & 0.800 & 0.873 & 6.723 & 0.060 \\
\hline SS19 & ef & eg & ee:ef:eg:fg & $1: 1: 1: 1$ & $44: 39: 42: 40$ & 0.4 & 21 & 0.476 & 0.617 & 2.513 & 0.209 \\
\hline SS20 & $\mathrm{nn}$ & np & $\mathrm{nn}: \mathrm{np}$ & $1: 1$ & $60: 90$ & 6.0 & 21 & 0.667 & 0.637 & 2.641 & -0.073 \\
\hline SS21 & $a b$ & $\mathrm{~cd}$ & $\mathrm{ac}: \mathrm{ad}: \mathrm{bc}: \mathrm{bd}$ & $1: 1: 1: 1$ & $31: 37: 41: 35$ & 1.4 & 21 & 0.857 & 0.920 & 9.800 & 0.046 \\
\hline SS22 & $-*$ & - & - & - & - & - & 21 & 0.238 & 0.220 & 1.273 & -0.111 \\
\hline SS23 & - & - & - & - & - & - & 21 & 0.429 & 0.418 & 1.690 & -0.050 \\
\hline SS24 & $a b$ & $\mathrm{~cd}$ & $a c: a d: b c: b d$ & $1: 1: 1: 1$ & $46: 53: 35: 51$ & 4.2 & 19 & 1.000 & 0.799 & 4.513 & -0.285 \\
\hline SS25 & $a b$ & $\mathrm{~cd}$ & $a c: a d: b c: b d$ & $1: 1: 1: 1$ & $34: 48: 32: 49$ & 6.0 & 20 & 0.950 & 0.958 & 15.094 & -0.017 \\
\hline SS26 & ef & eg & ee:ef:eg:fg & $1: 1: 1: 1$ & $39: 54: 41: 47$ & 3.0 & 21 & 0.667 & 0.826 & 5.158 & 0.173 \\
\hline SS27 & $\operatorname{lm}$ & 11 & $11: 1 \mathrm{~m}$ & $1: 1$ & $97: 92$ & 0.1 & 20 & 0.500 & 0.615 & 2.500 & 0.167 \\
\hline SS28 & $a b$ & $\mathrm{~cd}$ & ac: $a d: b c: b d$ & $1: 1: 1: 1$ & $49: 52: 42: 39$ & 2.4 & 20 & 0.700 & 0.897 & 8.000 & 0.200 \\
\hline SS29 & $a b$ & $\mathrm{~cd}$ & $\mathrm{ac}: \mathrm{ad}: \mathrm{bc}: \mathrm{bd}$ & $1: 1: 1: 1$ & $43: 43: 46: 53$ & 1.4 & 19 & 0.895 & 0.922 & 9.757 & 0.003 \\
\hline SS30 & $a b$ & $\mathrm{~cd}$ & $a c: a d: b c: b d$ & $1: 1: 1: 1$ & $39: 44: 50: 47$ & 1.5 & 18 & 0.667 & 0.724 & 3.375 & 0.053 \\
\hline SS31 & $a b$ & $\mathrm{~cd}$ & $a c: a d: b c: b d$ & $1: 1: 1: 1$ & $46: 49: 47: 49$ & 0.1 & 20 & 0.900 & 0.917 & 9.412 & -0.007 \\
\hline SS32 & $\operatorname{lm}$ & 11 & $11: 1 \mathrm{~m}$ & $1: 1$ & $78: 73$ & 0.2 & 21 & 1.000 & 0.858 & 6.168 & -0.194 \\
\hline SS33 & $\mathrm{nn}$ & $n p$ & $n n: n p$ & $1: 1$ & $90: 101$ & 0.6 & 21 & 0.524 & 0.610 & 2.471 & 0.120 \\
\hline SS34 & $\operatorname{lm}$ & 11 & $11: 1 \mathrm{~m}$ & $1: 1$ & $41: 37$ & 0.2 & 21 & 0.429 & 0.438 & 1.747 & -0.003 \\
\hline SS35 & $\mathrm{nn}$ & np & $\mathrm{nn}: \mathrm{np}$ & $1: 1$ & $99: 87$ & 0.8 & 19 & 0.421 & 0.679 & 2.947 & 0.363 \\
\hline SS36 & $\operatorname{lm}$ & 11 & $11: 1 \mathrm{~m}$ & $1: 1$ & $75: 107$ & 5.6 & 21 & 0.524 & 0.554 & 2.178 & 0.031 \\
\hline SS37 & - & - & - & - & - & - & 21 & 0.286 & 0.418 & 1.690 & 0.300 \\
\hline SS38 & $a b$ & $\mathrm{~cd}$ & $a c: a d: b c: b d$ & $1: 1: 1: 1$ & $37: 35: 34: 43$ & 1.3 & 20 & 0.600 & 0.664 & 2.837 & 0.073 \\
\hline SS39 & $a b$ & $\mathrm{~cd}$ & $a c: a d: b c: b d$ & $1: 1: 1: 1$ & $18: 20: 23: 21$ & 0.6 & 20 & 0.600 & 0.794 & 4.420 & 0.225 \\
\hline$S S 40$ & $\operatorname{lm}$ & 11 & $11: 1 \mathrm{~m}$ & $1: 1$ & $97: 89$ & 0.3 & 18 & 0.167 & 0.322 & 1.456 & 0.468 \\
\hline SS41 & - & - & - & - & - & - & 20 & 0.450 & 0.580 & 2.299 & 0.204 \\
\hline SS 42 & $a b$ & $\mathrm{~cd}$ & $a c: a d: b c: b d$ & $1: 1: 1: 1$ & $46: 45: 51: 49$ & 0.5 & 19 & 0.579 & 0.619 & 2.516 & 0.039 \\
\hline SS43 & $a b$ & $\mathrm{~cd}$ & $a c: a d: b c: b d$ & $1: 1: 1: 1$ & $35: 41: 44: 55$ & 4.8 & 20 & 0.700 & 0.915 & 9.302 & 0.216 \\
\hline SS 44 & $\operatorname{lm}$ & 11 & $11: 1 \mathrm{~m}$ & $1: 1$ & $87: 94$ & 0.3 & 21 & 0.286 & 0.441 & 1.757 & 0.337 \\
\hline SS 45 & $a b$ & $\mathrm{~cd}$ & $a c: a d: b c: b d$ & $1: 1: 1: 1$ & $48: 38: 35: 48$ & 3.2 & 21 & 0.857 & 0.836 & 5.444 & -0.050 \\
\hline SS 46 & $\operatorname{lm}$ & 11 & $11: 1 \mathrm{~m}$ & $1: 1$ & $78: 83$ & 0.2 & 20 & 0.700 & 0.683 & 2.996 & -0.051 \\
\hline SS47 & $a b$ & $\mathrm{~cd}$ & $a c: a d: b c: b d$ & $1: 1: 1: 1$ & $26: 37: 33: 32$ & 1.9 & 20 & 1.000 & 0.922 & 9.877 & -0.113 \\
\hline $\operatorname{SS} 48$ & - & - & - & - & - & - & 21 & 0.571 & 0.677 & 2.950 & 0.136 \\
\hline SS 49 & $a b$ & $\mathrm{~cd}$ & ac: $a d: b c: b d$ & $1: 1: 1: 1$ & $41: 40: 46: 39$ & 0.7 & 20 & 0.750 & 0.886 & 7.339 & 0.132 \\
\hline SS50 & $\operatorname{lm}$ & 11 & $11: 1 \mathrm{~m}$ & $1: 1$ & $28: 20$ & 0.7 & 20 & 0.700 & 0.651 & 2.740 & -0.102 \\
\hline SS51 & nn & $\mathrm{np}$ & $n n: n p$ & $1: 1$ & $68: 82$ & 1.3 & 21 & 0.857 & 0.899 & 8.167 & 0.023 \\
\hline SS52 & ef & eg & ee:ef:eg:fg & $1: 1: 1: 1$ & $49: 39: 34: 49$ & 4.0 & 21 & 0.667 & 0.841 & 5.582 & 0.188 \\
\hline SS53 & $a b$ & $\mathrm{~cd}$ & $a c: a d: b c: b d$ & $1: 1: 1: 1$ & $16: 21: 24: 23$ & 0.6 & 19 & 0.579 & 0.787 & 4.272 & 0.244 \\
\hline SS54 & - & - & - & - & - & - & 21 & 0.333 & 0.292 & 1.398 & -0.171 \\
\hline SS55 & $a b$ & $\mathrm{~cd}$ & $a c: a d: b c: b d$ & $1: 1: 1: 1$ & $46: 41: 42: 38$ & 0.8 & 20 & 0.650 & 0.755 & 3.792 & 0.117 \\
\hline SS56 & $\mathrm{nn}$ & $n p$ & $\mathrm{nn}: \mathrm{np}$ & $1: 1$ & $96: 89$ & 0.3 & 20 & 0.600 & 0.623 & 2.548 & 0.012 \\
\hline SS57 & $a b$ & $\mathrm{~cd}$ & $a c: a d: b c: b d$ & $1: 1: 1: 1$ & $27: 20: 28: 19$ & 0.4 & 21 & 0.762 & 0.820 & 5.011 & 0.048 \\
\hline $\begin{array}{l}\text { Mean } \\
\text { S.D. }\end{array}$ & & & & & & & 20 & $\begin{array}{l}0.633 \\
0.220\end{array}$ & $\begin{array}{l}0.699 \\
0.196\end{array}$ & $\begin{array}{l}4.659 \\
3.183\end{array}$ & \\
\hline
\end{tabular}

* A hyphen (-) indicates that the controlled cross was not polymorphic for that particular locus. Nomenclature for parental genotypes is adopted from JoinMap software package (http://www.kyazma.nl). 
The Mendelian segregation of the loci was examined by applying the chi-square test to data from the full-sib progeny.

The best fit protein was investigated by carrying out a blastx search in the NCBI website using default parameters.

All the markers presented in this paper have been checked against previously published microsatellite based EST markers in Picea to ensure there was no duplication.

\section{Results and Discussion}

Table 1 reports the PCR primer pairs used to amplify each locus, and the allele size range, as recorded from a panel of trees selected from the FR Sitka spruce breeding population situated at the Glencorse clone bank, near Edinburgh, UK (see LEE, 2001 for details). Variation in both perfect and imperfect repeat motifs was investigated. The most common class of data-mined dinucleotide repeat was AT, and this is in agreement with the findings of RUNGIS et al. (2004). The best protein match for each sequence was explored via the blastx function in Genbank, and the accessions showed matches from several kingdoms. The segregation ratios of all the loci were in accordance with a simple co-dominant mode of inheritance (Table 2). None of the loci deviated significantly from expected Mendelian segregations. The average number of alleles per locus in the panel of unrelated trees was 7.6 , with a range of $2-16$ alleles. This highlights that EST derived microsatellites are a good source of polymorphic markers. Indeed, RUNGIS et al. (2004) reported only a slightly higher average number of alleles in Sitka spruce gSSRs when compared to EST derived microsatellites (5.76/5.00). Observed heterozygosity varied considerably between loci, ranging from 0.17 to 1.00 (Table 2) with some evidence of null alleles based on $\mathrm{F}$ values and from the segregation data in the full-sib family. Within one of the full-sib family, six loci were monomorphic and 17 loci displayed four alleles and were therefore fully informative in this cross (Table 2). Forest Research aim to carry out a similar study in Sitka spruce to that of DEVEY et al. (2004), who explored the association between 92 co-dominant markers and economically important traits in Pinus radiata. A number of microsatellite markers are available in the literature for Picea, derived both from genomic and EST libraries. Sixteen markers specifically developed for Sitka spruce are published (VAN DE VEN and MCNICOL, 1996; A'HARA and CoTTRELL, 2004). In addition, a number of other studies within Picea report successful amplification in Sitka spruce, demonstrating the transferability of microsatellite markers (HODGETTS et al., 2001; RAJORA et al., 2001; RunGIS et al., 2004). However, to ensure a good framework map of Sitka spruce with a high degree of transferability across species this suite of 40 further co-dominant microsatellite markers is a significant addition to the Picea genetic mapping effort.

\section{Acknowledgements}

We are grateful to ANDRew PeAce, Biometrics Division, Forest Research, for statistical support.

\section{References}

A'Hara, S. W. and J. E. Cottrell (2004): A set of microsatellite markers for use in Sitka spruce (Picea sitchensis) developed from Picea glauca ESTs Molecular Ecology Notes 4: 659-663.

Devey, M. E., S. D. Carson, M. F. Nolan, A. C. Matheson, C. TE RIINI and J. HoHePA (2004): QTL associations for density and diameter in Pinus radiata and the potential for marker-aided selection. Theoretical \& Applied Genetics 108: 516-524.

Hodgetts, R. B., M. A. Aleksiuk, A. Brown, C. Clarke, E. MacDonald, S. NADEem and D. Khasa (2001): Development of microsatellite markers for white spruce (Picea glauca) and related species. Theoretical and Applied Genetics 102: 1252-1258.

HUANG, X. and A. MADAN (1999): CAP3: A DNA Sequence Assembly Program. Genome Research 9: 868-877.

Kolpakov, R., G. BANA and G. Kucherov (2003): mreps: efficient and flexible detection of tandem repeats in DNA. Nucleic Acids Research 31: 3672-3678.

LEE, S. J. (2001): Selection of parents for the Sitka spruce breeding population in Britain and the strategy for the next breeding cycle. Forestry 74, 129-143.

Liewlaksaneeyanawin, C., C. E. Ritland, Y. A. El-KassaBY and K. RITLAND (2004): Single-copy, species-transferable microsatellite markers developed from loblolly pine ESTs. Theoretical and Applied Genetics 109: 361-369.

RAJORA, O. P., M. H. RAHMAN, S. DAYANANDAN and A. Mosseler (2001): Isolation, characterisation, inheritance and linkage of microsatellite DNA markers in white spruce (Picea glauca) and their usefulness in other spruce species. Molecular and General Genetics 264: 871-882.

Rozen, S. and H. J. Skaletsky (2000): PRIMER3 on the www for general users and biologist programmers. In: Bioinformatics Methods and Protocols: Methods in Molecular Biology (eds. KRAwetz, S., Misener, S.), pp. 365-386. Humana Press, Totowa, NJ.

Rungis, D., Y. Berube, J. Zhang, S. Ralph, C. E. Ritland, B. E. Ellis, C. Douglas, J. Bohlmann and K. Ritland (2004): Robust simple sequence repeat markers for spruce (Picea spp.) from expressed sequence tags. Theoretical and Applied Genetics 109: 1283-94.

Ujino-Ihara, T., H. KanAMORI, H. YAmane, Y. TAGUChI, N. NAmiki, Y. MukaI, K. Yoshimura, Y. Tsumura (2005): Comparative analysis of expressed sequence tags of conifers and angiosperms reveals sequences specifically conserved in conifers. Plant Molecular Biology 59: 895-907.

VAN DE VEN, W. T. G. and R. J. MCNicol (1996): Microsatellites as DNA markers in Sitka spruce. Theoretical and Applied Genetics 93: 613-617.

YEH, F. C. and T. J. B. BoYLE (1997): Population genetic analysis of co-dominant and dominant markers and quantitative traits. Belgian Journal of Botany 129: 157. 